2018-06

\title{
Transient alpha and beta synchrony underlies preparatory recruitment of directional motor networks
}

\section{Rhodes, E}

http://hdl.handle.net/10026.1/10793

\author{
10.1162/jocn_a_01250 \\ Journal of Cognitive Neuroscience \\ Massachusetts Institute of Technology Press (MIT Press)
}

All content in PEARL is protected by copyright law. Author manuscripts are made available in accordance with publisher policies. Please cite only the published version using the details provided on the item record or document. In the absence of an open licence (e.g. Creative Commons), permissions for further reuse of content should be sought from the publisher or author. 
Journal of

Cognitive Neuroscience

\section{Transient alpha and beta synchrony underlies preparatory recruitment of directional motor networks}

\begin{tabular}{|r|l|}
\hline Journal: & Journal of Cognitive Neuroscience \\
\hline Manuscript ID & JOCN-2017-0367.R1 \\
\hline Manuscript Type: & Original \\
\hline Date Submitted by the Author: & 20 -Dec-2017 \\
\hline Complete List of Authors: & $\begin{array}{l}\text { Rhodes, Edward; University of Plymouth, School of Psychology } \\
\text { Marsden, Jonathan; University of Plymouth, School of Health Professions } \\
\text { Gaetz, William; Children's Hospital of Radiology, Radiology } \\
\text { Hall, Stephen; University of Plymouth, School of Psychology }\end{array}$ \\
\hline Keywords: & Motor control, EEG, Neural Oscillations, Somatosensory systems \\
\hline \multicolumn{2}{|l}{} \\
\hline
\end{tabular}


1

2

3

4

5

6

7

8

9

10

Transient alpha and beta synchrony underlies preparatory recruitment of directional motor networks.

Abbreviated Title: Alpha and Beta in recruitment of directional networks

Edward Rhodes ${ }^{1}$, Dr William C Gaetz ${ }^{2}$, Prof Jonathan Marsden ${ }^{3}$ \& Dr Stephen Hall ${ }^{1}$

1. School of Psychology, University of Plymouth, Devon, PL4 8AA, UK

2. Children's Hospital of Philadelphia, Philadelphia, PA, 19104, USA

3. School of Health Professions, University of Plymouth, Devon, PL4 8AA, UK

Corresponding Author: Edward Rhodes, Room 209, Link Building, Plymouth University, Plymouth, PL4 8AA, 01752 583021- edward.rhodes@plymouth.ac.uk

Pages: 18

Figures: 5

Tables: 0

\author{
Abstract: 206 words \\ Introduction: 802 words \\ Discussion: 987 words
}

The authors declare no competing financial interests. The authors would like to acknowledge (Funding Sources).

\title{
Acknowledgements
}

The authors of this work would like to thank Mr Martyn Atkins and Miss Anna Kharko for their technical support and assistance in collecting data for the present study. 
Abstract

Modulations in motor cortical beta and alpha activity have been implicated in the preparation, execution and termination of voluntary movements. The functional role of motor cortex beta activity is yet to be defined, though two opposing theories prevail. The idling cortex theory suggests that large-scale motor networks, in the absence of input, revert to an intrinsic oscillatory state. The alternative theory proposes that beta activity promotes postural tone at the expense of voluntary movement. These theories are primarily based upon observations of event-related desynchronisation associated with movement onset. Here, we explore the changes in alpha and beta oscillatory activity, associated with the specific behavioural patterns during an established directional uncertainty paradigm. We demonstrate that, consistent with current proposals, alpha and beta desynchronisation reflects a process of disengagement from existing networks, to enable the creation of functional assemblies. We demonstrate that following desynchronisation a novel signature, of transient alpha synchrony, underlies the recruitment of functional assemblies required for directional control. While alpha and beta desynchronisation are dependent upon the number of cues presented, they are not predictive of movement preparation. However, the transient alpha synchrony occurs only when participants have sufficient information to prepare for movement and shows a direct relationship with behavioural performance measures (reaction time). 


\section{Introduction}

Neuronal populations have intrinsic oscillatory properties that allow the synchronous activation of multiple networks within the brain. The primary motor cortex exhibits oscillatory activity within beta ( 13-30Hz) and alpha ( 8-12Hz) frequency bands (Pfurtscheller and Aranibar, 1979; Murthy and Fetz, 1992; Baker et al., 1997; Pfurtscheller, 1998). Motor-related beta activity is not constrained to the primary motor cortex (M1) and has been measured throughout the motor-related brain network (Klostermann et al., 2007). The cortical origin of the sensorimotor alpha (or $\mathrm{mu}$ ) and beta rhythms have been proposed to originate from separate sources, with beta generated in M1 (Baker, Olivier, \& Lemon, 1997; Murthy \& Fetz, 1992) and mu generated in primary somatosensory cortex (S1) (Salmelin and Hari 1994). However, recent studies demonstrate that both alpha and beta rhythms are generated in multiple laminae of M1 (Rönnqvist et al., 2013; Yamawaki et al., 2008), with amplitude dependent upon connectivity with S1 and other areas.

Intrinsic beta activity is modulated during the preparation, execution and termination of voluntary movements (Cheyne et al., 2008). Alterations in the modulation of beta have been associated with neuropathologies that exhibit motor abnormality, such as Parkinson's disease (PD) (Brown, 2003; Brown et al., 2004, Kuhn et al., 2006; Hall et al., 2014) and stroke (Tecchio et al., 2006; Hall et al., 2010).

The functional role of beta oscillatory activity is, as yet, unclear. However, evidence from local field potential (LFP) measures of the basal ganglia (BG) of PD patients suggests a link between exaggerated beta activity and impaired motor function (Brown and Williams, 2005; Kühn et al.,2005; Weinberger et al., 2006). Two theories have been suggested to explain the role of beta activity in motor function. The first, proposed by Pfurtscheller and colleagues (1996), suggests that beta activity is a correlate of idling motor activity. More recently, a second theory has proposed that beta activity promotes postural and tonic activity at the expense of voluntary movements (Gilbertson et al., 2005; Pastötter et al., 2008). Slowing of voluntary movement during intrinsic elevations in 
cortical beta activity (Gilbertson et al., 2005) and during $20 \mathrm{~Hz}$ entrainment using transcranial alternating current stimulation (tACS) of motor cortex (Pogosyan et al., 2009) has been cited as further evidence for this theory (Jenkinson and Brown, 2011).

Recording from implanted deep brain stimulation (DBS) electrodes enables measurement of LFPs, and therefore oscillatory activity, from the subthalamic nucleus (STN) of PD patients who suffer from increased tonic activity (rigidity) and a slowness of movement (bradykinesia). The observation of exaggerated beta power in the STN of these PD patients further supports the theory that M1 beta promotes tonic activity (Hammond et al., 2007) at the expense of voluntary movement. DBS to the STN suppresses beta activity and the degree of suppression correlates with the level of improvement in rigidity and bradykinesia (Kühn et al., 2008; Bronte-Stewart et al., 2009). This same correlation between the degree of reduced beta oscillatory activity and improvement in rigidity is observed following effective dopaminergic drug treatment (Kühn et al., 2006; Weinberger et al., 2006; Ray et al., 2008).

Further studies of both patient and neurotypical populations demonstrate beta suppression is a strong predictor of the efficacy of motor preparatory processes, with greater beta suppression reflected in shorter response times (Williams et al., 2003; Doyle, et al., 2005). The latency of motor response is well established to co-vary with the degree of certainty in the movement direction (Bock and Arnold, 1992; Dorris and Munoz, 1998; Pellizzer et al., 2006; Churchland et al., 2008). In the present study, we adopted an established motor-experimental paradigm for varying directional uncertainty (Pellizzer and Hedges, 2003; Pellizzer et al., 2006; Tzagarakis et al., 2010) to measure changes in beta oscillatory power. Previous studies observe that response times are significantly lengthened and beta ERD decreased in proportion to the number of cues (Tzagarakis et al., 2010). The aim of this study was to assess alpha and beta oscillatory power during each phase of the movement. Based upon the proposal that beta promotes tonic activity at the expense of voluntary movement and that preparatory beta suppression is a pre-requisite for movement onset, we 
hypothesised that, an increase in directional uncertainty will delay the termination of tonic activity, inhibit suppression of associated beta oscillatory activity and increase response times correspondingly. Furthermore, based upon the assumption that oscillatory networks represent transient functional assemblies, created for the purpose of temporally aligning relevant units to elicit an effective output. We hypothesised that following the initial uncoupling from the idling/tonic network, functional preparation would coincide with a transient increase in synchronous power. Here, we aimed to test the theory that the creation of a specific functional assembly, accompanied by a synchronous event, can only occur when the function (direction) is known. Therefore, it will occur in the relevant interval when the required direction of movement is revealed.

\section{Materials and Methods}

Subjects. Eighty right-handed volunteers (19 male), with a mean age of 27 (range $18-71$ ) were recruited. Informed consent was obtained and all studies were approved by the local ethics committee, in accordance with the ethical standards set by the 1964 Declaration of Helsinki.

Apparatus. Subjects were seated at a desk and gripped a custom-made joystick with their right hand, resting their forearm on the desk. The joystick was designed to be held between the thumb and forefinger and was capable of full $360^{\circ}$ rotation within the horizontal plane. The motor control task was displayed on a 21 -inch, high-definition monitor, placed at a comfortable height, $90 \mathrm{~cm}$ in front of the participant.

M1 Hand-area Localisation. Single-pulse transcranial magnetic stimulation (TMS) using a Magstim 2002 stimulator (Magstim, UK) and a standard 70mm diameter figure-of-eight coil held tangentially to the scalp with the coil handle pointing backward $\sim 45^{\circ}$ laterally. The electromyographic (EMG) activity of the first dorsal interosseous (FDI) muscle in the right hand was recorded using bipolar, single differential surface EMG electrodes (DE-2.1; Delsys). The surface electrodes comprised two 10 $1 \mathrm{~mm}$ silver bar strips, spaced $10 \mathrm{~mm}$ apart, with a $20 \mathrm{~Hz}-450 \mathrm{kHz}$ bandwidth, $92 \mathrm{~dB}$ common mode 
rejection ratio, and $<10^{15} \Omega$ input impedance. The electrodes were placed over the FDI muscle and a reference ground electrode was placed over the ulnar process of the right wrist. The EMG signal was digitised with a sampling rate of $2 \mathrm{kHz}$ using a Power 1401 digital-analogue (Cambridge Electronic Design, UK) converter and analysed using Signal version 6.04 (Cambridge Electronic Design, UK). The scalp location that elicited the greatest MEP response from the FDI muscle was designated as the primary hand area of left M1 and marked for positioning of the central recording site for the EEG montage.

Motor Control Task. The task used was an instructed-delay reaching task based on the established paradigm (Pellizzer and Hedges, 2003; Pellizzer et al., 2006; Tzagarakis et al., 2010) as seen in Figure 1. The task consisted of 180 trials, each comprising of a centre-hold period, a spatial cue presentation ('cue onset'), a target cue presentation ('target identification') and a participant response ('movement onset'). Each trial was initiated by the participant maintaining a joystickcontrolled crosshair within an outline of a circle in the centre of the screen (radius, $0.6^{\circ}$ of the visual angle (VA)) for 2s. Participants were instructed to fixate on the centre of the screen throughout the trial. Following the centre-hold period, a spatial cue was presented that varied randomly in duration between 1 and $1.5 \mathrm{~s}$. The spatial cue consisted of 1,2 or 3 possible targets, presented with the number and positions of targets pseudo-randomised for each trial. This resulted in 3 possible target presentations, each presented 20 times during the one target condition and 3 possible combinations of targets, each presented 20 times, during the two-target condition. Sixty trials in total were presented for each target condition. Each target consisted of an outline of a circle (radius, $0.75^{\circ} \mathrm{VA}$ ) with a centre $4^{\circ} \mathrm{VA}$ away from the centre of the screen. Targets were presented at $45^{\circ}, 165^{\circ}$ and $285^{\circ}$ relative to the centre of the screen. Following cue onset one of the targets was highlighted white (target identification). At this point, the participant used the joystick to move the crosshair from the centre of the screen towards the highlighted target circle as quickly and accurately as possible. Consequently, directional preparation was possible at cue onset in only the one-target 
condition, but not in the two-target or three-target conditions, where directional uncertainty continues until target identification.

The response time (RT) was defined as the period between a target identification and movement onset. For each of the three target conditions, RT outliers (median RT +/-2 MAD) were removed from the dataset. The task was controlled by a custom-made computer program (Microsoft Visual Basic).

EEG Recording and Analysis. A five electrode EEG montage was centred at the location of primary hand area of left M1. Four further electrodes were sited $2 \mathrm{~cm}$ anterior, posterior, ventral and dorsal with respect to the central electrode (Figure 1B). These electrodes were used to confirm the optimal spatial positioning of the $\mathrm{M} 1$ electrode. The $\mathrm{Ag} / \mathrm{AgCl}$ electrodes were coated with conductive Ten20 paste (Weaver and Company, USA) for 15 min before being affixed to the scalp, to ensure stable polarisation potential of the electrodes and reduce impedance. EEG recordings were made using a DC-EEG feedback NEURO PRAX system (neuroConn GmbH, Germany), referenced online to an electrode placed on the right mastoid process and sampled at $2048 \mathrm{~Hz}$ for digital storage.

\section{FIGURE 1 HERE}

EEG data from the recording montage were bandpass filtered between $2 \mathrm{~Hz}$ and a $100 \mathrm{~Hz}$ using a Hamming window-synced FIR filter (Oppenheim, Schafer \& Buck,1999) and notch-filtered at $50 \mathrm{~Hz}$ to reduce electrical mains noise. All data were processed using Matlab and FieldTrip open-source Matlab toolbox (Oostenveld et al., 2011). Time-frequency analysis was performed using a Morlet wavelet transformation (Bertrand and Pantev, 1994; Tallon-Baudry and Bertrand, 1999). Spectral power in the alpha $(8-12 \mathrm{~Hz})$ and beta $(13-30 \mathrm{~Hz})$ bands were analysed based upon the mean amplitude in each band. Analysis of resting beta $(13-30 \mathrm{~Hz})$ power, during an initial 2-minute recording, was used to confirm the central electrode as the optimal location for further analysis, which was used for all subsequent analysis. Epochs were defined using digital triggers produced by the EEG system for both cue onset and target identification for each trial. The preparation stage of 
movement was defined as the phase between cue onset and target identification for each trial (1000-1500ms). Successful trials were defined as trials in which the participant identified the correct target and moved the crosshair to the centre of the target circle. Unsuccessful trials were removed from analysis along with any trial in which the signal to noise ratio was too low. Subjects were removed from the analysis if over $20 \%$ of their trials were lost. As a result, the final analysis was carried out on 74 out of 80 subjects.

\section{Experimental Design and Statistical Analysis}

A fully counterbalanced repeated measures design was used. Differences in response times, beta and alpha amplitude across the 3 directional uncertainty conditions were analysed using analyses of variance (ANOVAs) and paired t-tests. The latency of oscillatory power change (event-related desynchronisation ) was identified as the time point at which oscillatory power fell 2.5SD from the mean baseline power, following the method previously described (McAllister et al., 2013). Change in beta power was analysed with respect to baseline $500 \mathrm{~ms}$ before cue onset, for the initial change following cue onset and target identification. To further determine the relative change in oscillatory power between target identification and movement onset, data were normalised to the point of target identification.

To test the hypothesis that creation of a functional assembly requires, and therefore coincides, with transient synchrony. We analysed the change in synchronous power following the initial desynchronisation (uncoupling) from the beta. We used a sliding window approach (50ms) to compute the change in synchronous power between conditions $(1,2$, and 3 Targets) from the minimum following cue onset to the maximum in the interval up to target identification. We used the same approach to determine the difference in power between the $100 \mathrm{~ms}$ pre-target identification and the interval up to movement onset. This approach was used to identify differences in synchronous power when direction was known in the post-cue onset (1 Target) or post-target identification (3 Target) conditions. 


\section{Results}

\section{Behavioural results}

Average RT are plotted against number of targets in Figure 1C. Results from a one-way ANOVA indicated that RT was significantly affected by number of targets $\left(F_{(2,148)}=488.84, p<0.001\right.$, ANOVA), with planned contrasts indicating that RT during 1-target presentation $(M=372.1 \mathrm{~ms}, \mathrm{SD}=56.2 \mathrm{~ms})$ were significantly less than during 2-target presentation $\left(M=427.8 m s, S D=55.6 m s, t_{(73)}=-21.84\right.$, $p<0.001$, paired t-test) and 3-target presentation $\left(M=449.5 \mathrm{~ms}, S D=57.5 \mathrm{~ms}, t_{(73)}=-30.3, p<0.001\right.$, paired t-test). Average RT during 2-target presentation was also significantly lower than during 3target presentations $\left(t_{(73)}=-8.46, p<0.001\right.$, paired t-test). These findings are in line with those of previous studies using a similar paradigm that found as directional uncertainty increases response times lengthen (Pellizzer and Hedges, 2003; Pellizzer et al., 2006; Tzagarakis et al., 2010).

\section{Time-series analysis of beta ERD}

For each subject, beta-band power was normalised relative to a $1000 \mathrm{~ms}$ baseline period, starting $1500 \mathrm{~ms}$ prior to cue onset and averaged across all conditions. On a trial-by-trial basis, the power change from baseline to a $500 \mathrm{~ms}$ period after cue onset was computed to determine the initial preparatory beta ERD. For each participant, power change was averaged for the number of targets presented (Figure 2).

\section{FIGURE 2 HERE}

A decrease in beta-band power after cue onset was observed in all conditions (1, 2 and 3-targets). The onset-latency of this beta desynchronisation occurred on average $173.8 \mathrm{~ms}$ (SD=115.3ms) after cue onset with no significant difference between target conditions $\left(F_{(2,148)}=689, p=0.504\right.$, ANOVA $)$. The amplitude of initial preparatory beta desynchronisation was significantly different between 
conditions $\left(F_{(2,148)}=3.25, p=0.041\right)$, showing smaller reduction in power as the number of targets, and therefore the directional uncertainty, increased (Figure 3A). Specifically, during 1-target trials, where subjects were certain of the direction of movement required, a significantly greater beta desynchronisation $(M=20.25 \%, S D=20.47 \%)$ was observed than with 3 -target trials $(M=12.53 \%$, $S D=18.92 \%)$, where required direction was unknown $\left(t_{(73)}=-2.47, p=0.014\right.$, paired t-test, Figure 3B).

Following the initial preparatory desynchronisation, the 1-target condition exhibited sustained beta suppression, whereas the 2-target and 3-target conditions showed a partial resynchronisation (i.e., an increase in beta power) until the point of target identification (Figure 3C).

Consequently, at the point of target identification, when directional uncertainty was resolved and subjects were cued to move, beta power was significantly greater in the 3-target $(M=83.14 \%$, $S D=18.97 \%)$ than the 1-target $(M=75.41 \%, S D=16.27 \%)$ condition $\left(t_{(73)}=-2.84, p=0.005\right.$, paired t-test, Figure 3D).

\section{FIGURE 3 HERE}

Following target identification, average beta power reduced in all conditions, until the point of movement initiation, termed 'response phase ERD' (Figure 4A). The latency of response phase ERD showed no dependence upon the number of targets presented $\left(F_{(2,148)}=1.035, p=0.358, A N O V A\right)$. In contrast with a previous observation (Tzagarakis et al. 2010), we observed that the amplitude of the response phase ERD was not significantly different between the three different target conditions $\left(F_{(2,148)}=.812, p=0.445\right.$, ANOVA) (Figure $\left.4 B\right)$. This can be observed from the envelope of beta power when normalised to the $500 \mathrm{~ms}$ prior to target identification (Figure $4 \mathrm{C}$ ).

FIGURE 4 HERE 


\section{Time-series analysis of alpha ERD}

Time-series analysis within the alpha frequency range $(8-12 \mathrm{~Hz})$, revealed a decrease in power in all conditions following initial cue onset (Figure 5A.). Alpha power showed significantly greater desynchronisation following 1-target (high directional certainty) preparation $(M=20.18 \% / S D=21.43 \%)$, compared to 2 -target $(M=10.14 \% / S D=29.55 \%)$ preparation $\left(t_{(73)}=-2.17\right.$, $\mathrm{p}=0.031$, paired $\mathrm{t}$-test $)$ and 3-target $(\mathrm{M}=12.63 \% / \mathrm{SD}=24.6 \%)$ preparation $\left(\mathrm{t}_{(148)}=-2.13, \mathrm{p}=0.034\right.$, paired t-test).

Following initial alpha response-phase ERD, a transient increase in alpha synchrony was observed in the 1-target condition $\left(\mathrm{M}=2.93 \%, \mathrm{SD}=13.04 \%, \mathrm{t}_{(73)}=1.936, \mathrm{p}=.057\right.$, paired $\mathrm{t}$-test), but not the 2-target $\left(M=-2.33 \%, S D=14.58 \%, t_{(73)}=-1.373, p=.174\right.$, paired $t$-test $)$ or $3-\operatorname{target}\left(M=-.73 \%, S D=14.93 \%, t_{(73)}=-\right.$ $.418, p=.677$, paired $t$-test) conditions (Figure $5 B$ ). Conversely, following target identification, a significant transient increase in alpha synchrony was observed in the 2-target $(M=6.32 \%$, $S D=17.34 \%, t_{(73)}=3.134, p=.002$, paired $t$-test $)$ and 3-target $\left(M=5.2 \%, S D=14.71 \%, t_{(73)}=3.039, p=.003\right.$, paired t-test) conditions, but not the 1-target $\left(\mathrm{M}=-.89 \%, \mathrm{SD}=12.38 \%, \mathrm{t}_{(73)}=-.617, \mathrm{p}=.539\right.$, paired $\mathrm{t}$ test) condition (Figure 5D). This condition-dependent alpha synchrony is clearly visible in the envelope of alpha power (Figure 5A \& C).

\section{FIGURE 5 HERE}

\section{Reaction Time and Transient Alpha Synchrony}

To further investigate the relationship between transient alpha synchrony and reaction time, we analysed the latency of peak alpha ERS following target identification in the 2 and 3-target conditions. We found that peak alpha synchrony occurred significantly earlier in the 2-target condition $(M=203.4 \mathrm{~ms}, S D=75.56 \mathrm{~ms})$ than in the 3 -target condition $(M=242.7 \mathrm{~ms}, S D=72.44 \mathrm{~ms}$, $F_{(2,148)}=5.525, p=0.021$, ANOVA), corresponding to the shorter reaction time in the 2-target condition. 


\section{Discussion}

The present study suggests that the profile of synchronous power change in the alpha and beta frequency range is directly associated with the recruitment of appropriate networks in preparation for movement.

As expected, reaction-time was directly dependent upon the ability of participants to predict the direction of required movement, based upon the stimulus information. This is consistent with previous reports (Bock and Arnold, 1992; Dorris and Munoz, 1998; Pellizzer et al., 2006; Churchland et al., 2008; Tzagarakis et al., 2010) and confirms that participants engaged with the experiment as required.

Consistent with general observations in a variety of experiments involving movement paradigms (Pfurtscheller and Aranibar, 1979; Pfurtscheller et al., 1996; Pfurtscheller and Lopes da Silva, 1999; Jurkiewicz et al., 2006; Cheyne, 2013), a significant decrease in both alpha and beta synchronous power was observed following the presentation of the initial onset cue in all conditions. We propose that this desynchrony reflects a process of disengagement from on-going network activity, to allow the assignment of appropriate motor units to generate a required output. Of note, the amplitude of both alpha and beta ERD is directly dependent upon the number of targets presented. This suggests that, where information is present that allows for the identification of the appropriate response, those neural populations are enabled to be disengaged in preparation for recruitment to an output assembly. Taken together, these observations of disengagement from an active network support the theory of beta, and indeed alpha, oscillations in the promotion of postural tone at the expense of voluntary movement (Gilbertson et al., 2005; Pastötter et al., 2008). However, the observation of partial resynchronisation of the beta rhythm in the two and three target conditions is also consistent with an inhibition of movement in the absence of a motor plan. Following initial ERD, we demonstrate that the on-going profile of beta power is predictive of the participant's preparatory state. In the 1-target condition, beta suppression is sustained up to the point of target identification, 
whereas the 2-target and 3-target conditions exhibit a partial resynchronisation of beta power until the required target direction is indicated. Given that temporary re-assignment to a postural network is unlikely, with the impending need to move, resynchronisation is likely to reflect the temporal realignment of potential units with the functional networks required to generate motor output after target identification. While speculative, this suggests that beta synchrony in motor cortex reflects and serves a dual purpose, including the maintenance of postural tone (Gilbertson et al., 2005; Pastotter et al., 2008) and providing a temporal pacemaker to maximise effective motor responses.

The precise role(s) of the beta rhythm notwithstanding, these data support the independence of the alpha and beta rhythms, which subserve separate functional processes see (van Wijk, 2012) for a review. Importantly, we identify a novel oscillatory feature in this experiment that corresponds to the participants' ability to engage a specific motor network. Following the initial alpha and beta ERD, a transient 'burst' of alpha is observed in the 1-target condition, but not in the 2-target or 3-target conditions. We propose that this signature reflects the recruitment of units to the functional assembly required to generate the motor output. This is supported by the observation that following target identification a transient burst of alpha is observed in the 2-target and 3-target conditions, but not in the 1-target condition. These signatures are temporally consistent with the participants' ability to assign the appropriate network, based upon information about the required movement. This feature dependency is borne-out behaviourally, as the alpha burst occurs significantly earlier in the 2-target than the 3-target condition, corresponding to the significant difference in reaction time between those conditions. This feature is consistent with electrophysiological recordings which have shown alpha oscillations reflect feedback inhibition in the cortex (Bastos et al., 2015; Michalareas et al., 2016), with feedback inhibition suggested to tune directional pyramidal cells in the motor cortex (Georgopoulos \& Stefanis, 2007; Merchant, Naselaris \& Georgopoulos, 2008; Isomura et al., 2009). We posit, given the occurrence of the transient alpha signal only when sufficient information is available to form a motor plan, that it reflects the recruitment in readiness of units required to execute the movement. This may include pyramidal tract neurons, but they are unlikely to be the 
sole generator given the relative minority of these cells (Keller, 1993). The switching of beta and alpha here, given the relatively low spatial resolution of the measurement, is consistent with gating by inhibition hypothesis (Jensen \& Mazaheri, 2010). In particular, when considered alongside the observation of increased alpha in M1, following removal of connectivity with S1 (Rönnqvist et al., 2013), it is possible that increased alpha reflects active inhibition of somatosensory input to facilitate formation of a motor plan based upon current information. Indeed, the inhibitory basis of these signals is supported by several studies that demonstrate the role of GABAergic modulation in generation of the beta (Hall et al., 2010; 2011; Jensen et al., 2005; Muthukumaraswamy et al., 2013) and alpha (Rönnqvist et al., 2013) rhythms.

In contrast with previous observations (Tzagarakis et al., 2010), we observed no significant difference between conditions in either the latency or the amplitude of the response-phase ERD. Importantly, the differences in response-phase ERD amplitude can be accounted for directly by the oscillatory power at the point of target identification, which is predicted by the number of targets. Further change in synchronous power is removed by normalisation to that point. We propose that, while beta desynchronisation is an important process in the generation of movement, the critical distinction in this experiment lies in the preparatory, rather than response, phase.

In summary, we demonstrate that both alpha and beta desynchronisation reflect a process of disengagement from existing networks, to enable the creation of functional assemblies. We also demonstrate a novel signature of transient alpha synchrony, which is predictive of reaction time and thus likely associated with the recruitment of a functional assembly required to generate the motor output. 


\section{References}

Baker, S. N., Olivier, E., \& Lemon, R. N. (1997). Coherent oscillations in monkey motor cortex and hand muscle EMG show task-dependent modulation. J Physiol, 501, 225-241.

Bastos, A. M., Vezoli, J., Bosman, C. A., Schoffelen, J. M., Oostenveld, R., Dowdall, J. R., . . Fries, H. (2015). Visual areas exert feedforward and feedback influences through distinct frequency channels. Neuron, 85(2), 390-401.

Bertrand, O., \& Pantev, C. (1994). Stimulus frequency dependence of the transient oscillatory auditory evoked response $(40 \mathrm{~Hz})$ studied by electric and magnetic recordings in human. In C. Pantev, T. Elbert, \& B. Lütkenhöner (Eds.), Oscillatory event-related brain dynamics (pp. 231-242). New York: Plenum Press.

Bock, O., \& Arnold, K. (1992). Motor control prior to movement onset: preparatory mechanisms for pointing at visual targets. Exp Brain Res, 90, 209-216.

Bronte-Stewart, H., Barberini, C., Koop, M. M., Mill, B. C., Henderson, J. M., \& Wingeier, B. (2009). The STN beta-band profile in Parkinson's disease is stationary and shows prolonged attenuation after deep brain stimulation. Experimental Neurology, 215(1), 20-28.

Brown, P. (2003). Oscillatory nature of human basal ganglia activity: relationship to the pathophysiology of Parkinson's disease. Movement Disorders, 357-363.

Brown, P., \& Williams, D. (2005). Basal ganglia local field potential activity: character and functional significance in the brain. Clin Neurophysiol, 116, 2510-2519.

Brown, P., Mazzone, P., Oliviero, A., Altibrandi, M. G., Pilato, F., Tonali, P. A., \& Di Lazzaro, V. (2004). Effects of stimulation of the subthalamic area on oscillatory pallidal activity in Parkinson's disease. Experimental Neurology, 188(2), 480-490.

Cheyne, D. O. (2013). MEG studies of sensorimotor rhythms: a review. Exp. Neurol., 245, 27-39. 
Cheyne, D., Bells, S., Ferrari, P., Gaetz, W., \& Bostan, A. C. (2008). Self-paced movements induce high-frequency gamma oscillations in primary motor cortex. Neuroimage, 42(1), 332-342.

Churchland, A. K., Kiani, R., \& Shadlen, M. N. (2008). Decision making with multiple alternatives. Nature Neuroscience, 11, 693-702.

Dorris, M. C., \& Munoz, D. P. (1998). Sacaadic probability influences motor preparation signals and time to saccadic initiation. Journal of Neuroscience, $18,7015-7026$.

Doyle, L. M., Yarrow, K., \& Brown, P. (2005). Lateralization of event-related beta desynchronization in the EEG during pre-cued reaction time tasks. Clin Neurophysiol, 116, 1879-1888.

Georgopoulos, A. P., \& Stefanis, C. N. (2007). Local shaping of function in the motor cortex: motor contrast, directional tuning. Brain research reviews, 55(2), 383-389.

Gilbertson, T., Lalo, E., Doyle, L., Di Lazzaro, V., Cioni, B., \& Brown, P. (2005). Existing motor state is favored at the expense of new movement during 13-35 Hz oscillatory synchrony in the human corticospinal system. Journal of Neuroscience, 25(34), 7771-7779.

Hall, S. D., Barnes, G. R., Furlong, P. L., Seri, S., \& Hillebrand, A. (2010). Neuronal Network Pharmacodynamics of GABAergic Modulation in the Human Cortex Determined Using Pharmaco-Magnetoencephalography. Human Brain Mapping, 31, 581-594.

Hall, S. D., Stanford, I. M., Yamawaki, N., McAllister, C. J., Rönnqvist, K. C., Woodhall, G. L., \& Furlong, P. L. (2011). The role of GABAergic modulation in motor function related neuronal network activity. Neurolmage, 56(3), 1506-1510

Hammond, C., Bergman, H., \& Brown, P. (2007). Pathological synchronization in Parkinson's disease: networks, models and treatments. Trends in Neuroscience, 30(7), 357-364. 
Isomura, Y., Harukuni, R., Takekawa, T., Aizawa, H., \& Fukai, T. (2009). Microcircuitry coordination of cortical motor information in self-initiation of voluntary movements. Nature Neuroscience, 12(12), 1586-93.

Jenkinson, N., \& Brown, P. (2011). New insights into the relationship between dopamine, beta oscillations and motor function. Trends in Neuroscience, 34(12), 611-618.

Jensen, O., \& Mazaheri, A. (2010). Shaping Functional Architecture by Oscillatory Alpha Activity: Gating by Inhibition. Frontiers in Human Neuroscience, 4.

Jensen, O., Goel, P., Kopell, N., Pohja, M., Hari, R., \& Ermentrout, B. (2005). On the human sensorimotor-cortex beta rhythm: sources and modeling. Neurolmage, 26(2), 347-355.

Jurkiewicz, M. T., Gaetz, W. C., Bostan, A. C., \& Cheyne, D. (2006). Post-movement beta rebound is generated in motor cortex: evidence from neuromagnetic recordings. Neurolmage, 32, 1281-1289.

Keller, A. (1993). Intrinsic Synaptic Organization of the Motor Cortex. Cerebral Cortex, 3(5), 430-441

Klostermann, F., Nikulin, V., Kühn, A. A., Marzinzik, F., Wahl, M., Pogosyan, A., . . Curio, G. (2007). Task-related differential dynamics of EEG alpha and beta-band synchronization in corticobasal motor structures. European Journal of Neuroscience, 25, 1604-1615.

Kühn, A. A., Kempf, F., Brücke, C., Doyle, L. G., Martinez-Torres, I., Pogosyan, A., . . Brown, P. (2008). High-frequency stimulation of the subthalamic nucleus suppresses oscillatory $\beta$ activity in patients with Parkinson's disease in parallel with improvement in motor performance. Journal of Neuroscience, 28(24), 6165-6173.

Kühn, A. A., Kupsch, A., Schneider, G. H., \& Brown, P. (2006). Reduction in subthalamic 8-35Hz oscillatory activity correlates with clinical improvement in Parkinson's disease. European Journal of Neuroscience, 23(7), 1956-1960. 
Kühn, A. A., Trottenberg, T., Kivi, A., Kupsch, A., Schneider, G. H., \& Brown, P. (2005). The relationship between local field potential and neuronal discharge in the subthalamic nucleus of patients with Parkinson's disease. Experimental neurology, 194(1), 212-220.

Merchant, H., Naselaris, T., \& Georgopoulos, A. P. (2008). Dynamic sculpting of directional tuning in the primate motor cortex during three-dimensional reaching. Journal of Neuroscience, 28(37), 9164-9172.

Michalareas, G., Vezoli, J., Van Pelt, S., Schoffelen, J. M., Kennedy, H., \& Fries, P. (2016). Alpha-beta and gamma rhythms subserve feedback and feedforward influences among human visual cortical areas. Neuron, 89(2), 384-397.

Murthy, V. N., \& Fetz, E. E. (1992). Coherent 25- to 35-Hz oscillations in the sensorimotor cortex of awake behaving monkeys. Proc Natl Acad Sci USA, 89, 5670-5674.

Muthukumaraswamy, S. D., Myers, J. F. M., Wilson, S. J., Nutt, D. J., Lingford-Hughes, A., Singh, K. D., \& Hamandi, K. (2013). The effects of elevated endogenous GABA levels on movementrelated network oscillations. Neurolmage, 66, 36-41.

Oostenveld, R., Fries, P., Maris, E., \& Schoffelen, J. M. (2010). FieldTrip: open source software for advanced analysis of MEG, EEG and invasive electrophysiological data. Computational intelligence and neuroscience(2011).

Pastötter, B., Haslmayr, S., \& Bäuml, K.-H. (2008). Inhibition of return arises from inhibition of response processes: an analysis of oscillatory beta activity. Journal of Cognitive Neuroscience, 20(1), 65-75.

Pellizzer, G., \& Hedges, J. H. (2003). Motor planning: effect of directional uncertainty with discrete spatial cues. Exp Brain Res, 150, 276-289. 
Pellizzer, G., Hedges, J. H., \& Villanueva, R. R. (2006). Time-dependent effects of discrete spatial cues on the planning of directed movements. Exp Brain Res, 172, 22-34.

Pfurtscheller, G. (1998). EEG event-related desynchronization (ERD) and event-related synchronization (ERS). In E. Niedermeyer, \& F. H. Lopes da Silva (Eds.), Electroencephalography: Basic Principles, Clinical Applications and Related Fields (pp. 958967). Baltimore: Williams and Wilkins.

Pfurtscheller, G., \& Aranibar, A. (1979). Evaluation of event-related desynchronization (ERD) preceding and following voluntary self-paced movement. Electroencephalography and Clinical Neurophisiology, 46, 138-146.

Pfurtscheller, G., \& Lopes da Silva, F. H. (1999). Event-related EEG/MEG synchronization and desynchronization: basic principles. Clinical Neurophysiology, 98, 281-293.

Pfurtscheller, G., Stancák Jr., A., \& Neuper, C. (1996). Post-movement beta synchronization. A correlate of an idling motor area? Electroencephalography and clinical neurophysiology, 98, 281-293.

Pogosyan, A., Gaynor, L. D., Eusebio, A., \& Brown, P. (2009). Boosting cortical activity at beta-band frequencies slows movement in humans. Current Biology, 19, 1637-1641.

Ray, N., Jenkinson, N., Wang, S., Holland, P., Brittain, J. S., Joint, C., . . Aziz, T. (2008). Local field potential beta activity in the subthalamic nucleus of patients with Parkinson's disease is associated with improvements in bradykinesia after dopamine and deep brain stimulation. Experimental Neurology, 213, 108-113.

Rönnqvist, K. C., McAllister, C. J., Woodhall, G. L., Stanford, I., \& Hall, S. D. (2013). A multimodal perspective on the composition of cortical oscillations. Frontiers in Human Neuroscience, 7(132). 
Tallon-Baudry, C., \& Bertrand, O. (1999). Oscillatory gamma activity in humans and its role in object representation. Trends in Computer Science, 3, 151-161.

Tecchio, F., Zappasodi, F., Tombini, M., Oliviero, A., Pasqualetti, P., Vernieri, F., . . Rossini, P. M. (2006). Brain plasticity in recovery from stroke: an MEG assessment. Neuroimage, 13261334.

Tzagarakis, C., Ince, N. F., Leuthold, A. C., \& Pellizzer, G. (2010). Beta-band activity during motor planning reflects response uncertainty. Journal of Neuroscience, 30(34), 11270-11277.

Tzagarakis, C., West, S., \& Pellizzer, G. (2015). Brain oscillatory activity during motor preparation: effect of directional uncertainty on beta, but not alpha, frequency band. Frontiers in Neuroscience, 9(246).

Weinberger, M., Mahant, N., Hutchinson, W. D., Lozano, A. M., Moro, E., Hodaie, M., Dostrovsky, J. O. (2006). Beta oscillatory activity in the subthalamic nucleus and its relation to dopaminergic response in Parkinson's disease. Journal of Neurophysiology, 96(6), 32483256.

Williams, D., Kühn, A. A., Kupsch, A., Tijssen, M., van Bruggen, G., Speelman, H., . . Brown, P. (2003). Behavioural cues are associated with modulations of synchronous oscillations in the human subthalamic nucleus. Brain, 126, 1975-1985.

van Wijk, B. C. M. (2012). Neural synchrony within the motor system: what have we learned so far? Frontiers in Human Neuroscience. 6 (252), 1-15.

Yamawaki, N., Stanford, I. M., Hall, S. D., \& Woodhall, G. L. (2008). Pharmacologically induced and stimulus evoked rhythmic neuronal oscillatory activity in the primary motor cortex in vitro. Neuroscience, 151(2), 386-395. 


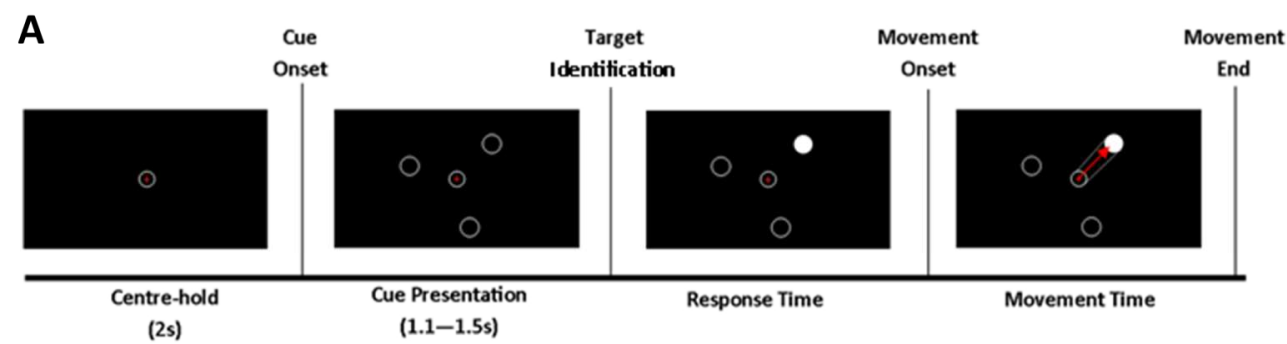

B

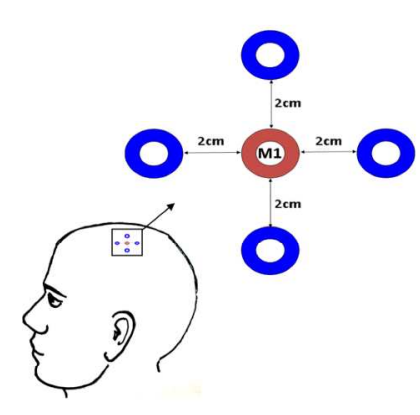

C

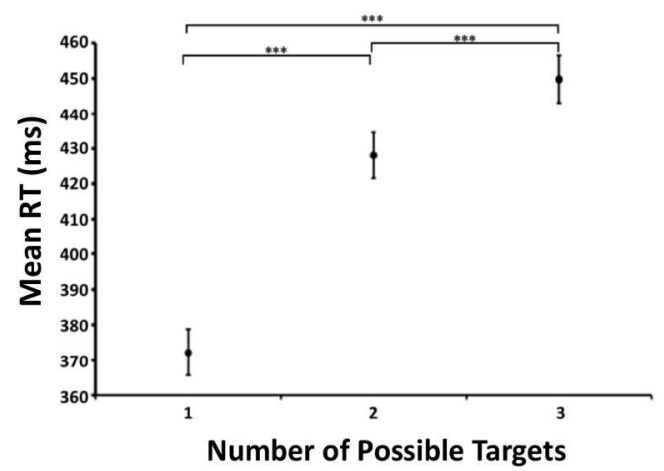

Figure 1. (A) Diagram of the trial sequence for the instructed-delay task. Participants used the joystick to hold the crosshair in the centre of the screen for $2 \mathrm{~s}$. Then 1, 2 or 3 potential targets were presented on the screen for $1.1-1.5 \mathrm{~s}$. The figure depicts a '3 Target' trial in which the target at 45 degrees was highlighted as the target the participant was to move the crosshair toward. RT was defined as the duration between target identification and movement onset. (B) Schematic showing the EEG electrode montage, centred over primary motor cortex, as defined using TMS motor localisation. (C) Average RT for each number of target conditions. The error bars represent standard error $(\mathrm{N}=74$ Subjects). 1 Target $(M=372.16 \mathrm{~ms}), 2$ Targets $(\mathrm{M}=428.09 \mathrm{~ms})$ and 3 Targets $(M=449.78 \mathrm{~ms})$. 


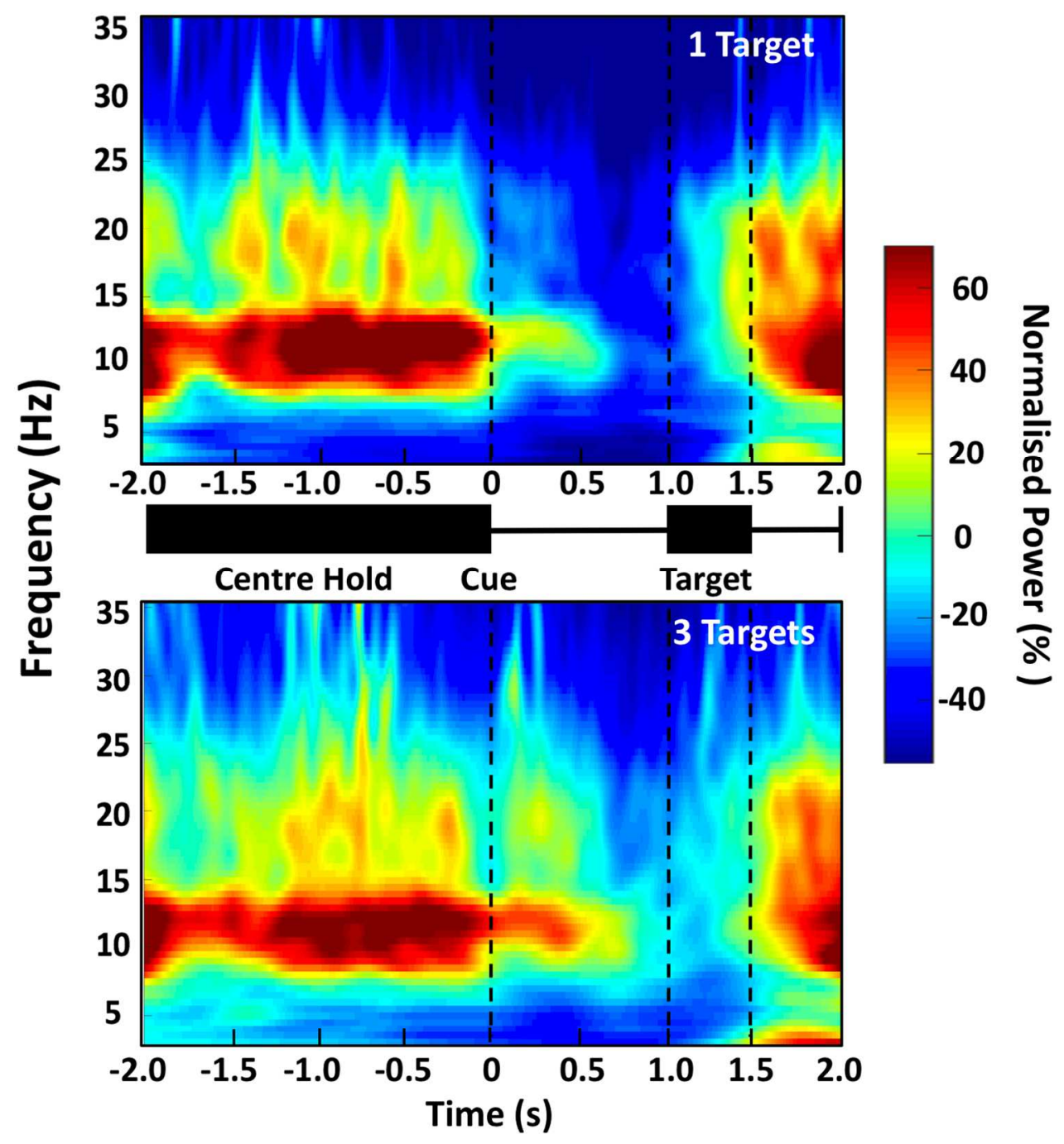

Figure 2. Averaged time-frequency power plots across participants for 1 and 3 Target conditions. The central schematic shows the 'centre hold' period (2s), the 'cue onset' and 'target' onset intervals. The timefrequency power plots show the profile of oscillatory power change (\%) with respect to the pre-stimulus baseline period. A greater reduction in beta-band $(13-30 \mathrm{~Hz})$ power is associated with movement preparation afforded by directional certainty (1-target) compared with directional uncertainty (3-targets).

$$
265 \times 280 \mathrm{~mm}(144 \times 144 \mathrm{DPI})
$$




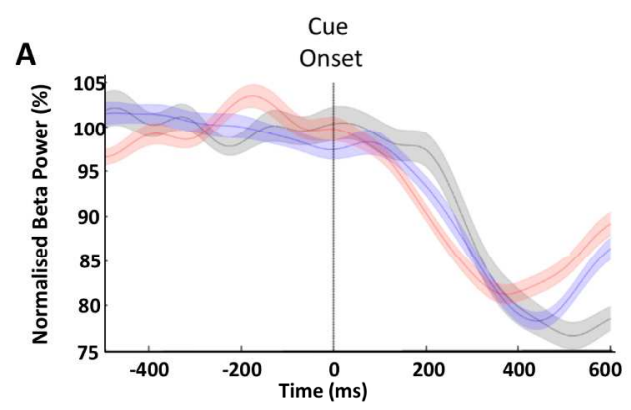

\section{B}

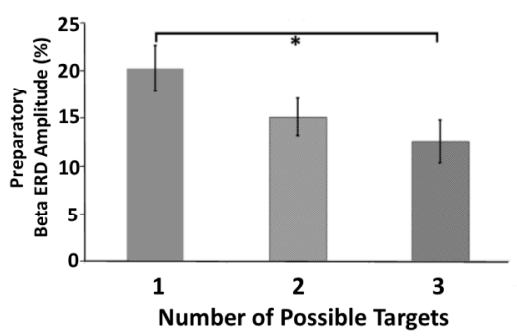

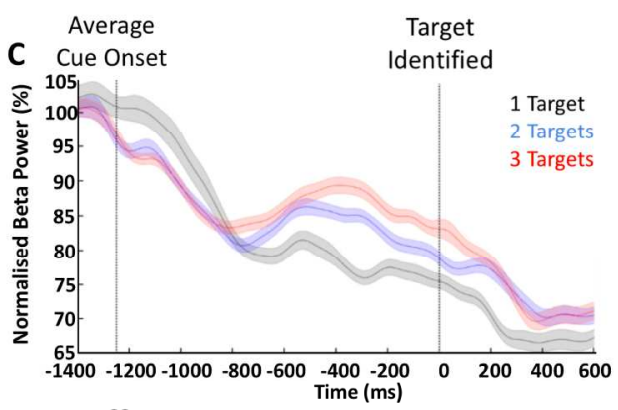

D

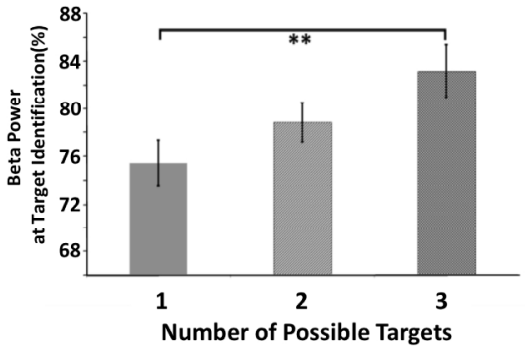

Figure 3. Power envelope plots of normalised preparatory beta power for each of the three target conditions (mean +/-SEM). (A) Initial changes in preparatory beta power after cue onset. The onset latency of beta ERD ( $<2.5 S D$ of baseline) does not vary across conditions, however, the absolute reduction in beta power is greater in the 1-target condition than 2 or 3-target conditions. (B) Bar chart showing the amplitude of initial beta ERD (mean +/-SD). Beta desynchronisation is significantly greater in the 1 target than the 3-target condition $(p=.014)$. C. Reduced Beta power is sustained up to the point of target identification the 1-target condition. The 2 and 3-target conditions show a partial resynchronisation in this interval. (D) Beta power is significantly greater at the point of target identification during 3 target, than 1 target conditions $(p=.005)$. 

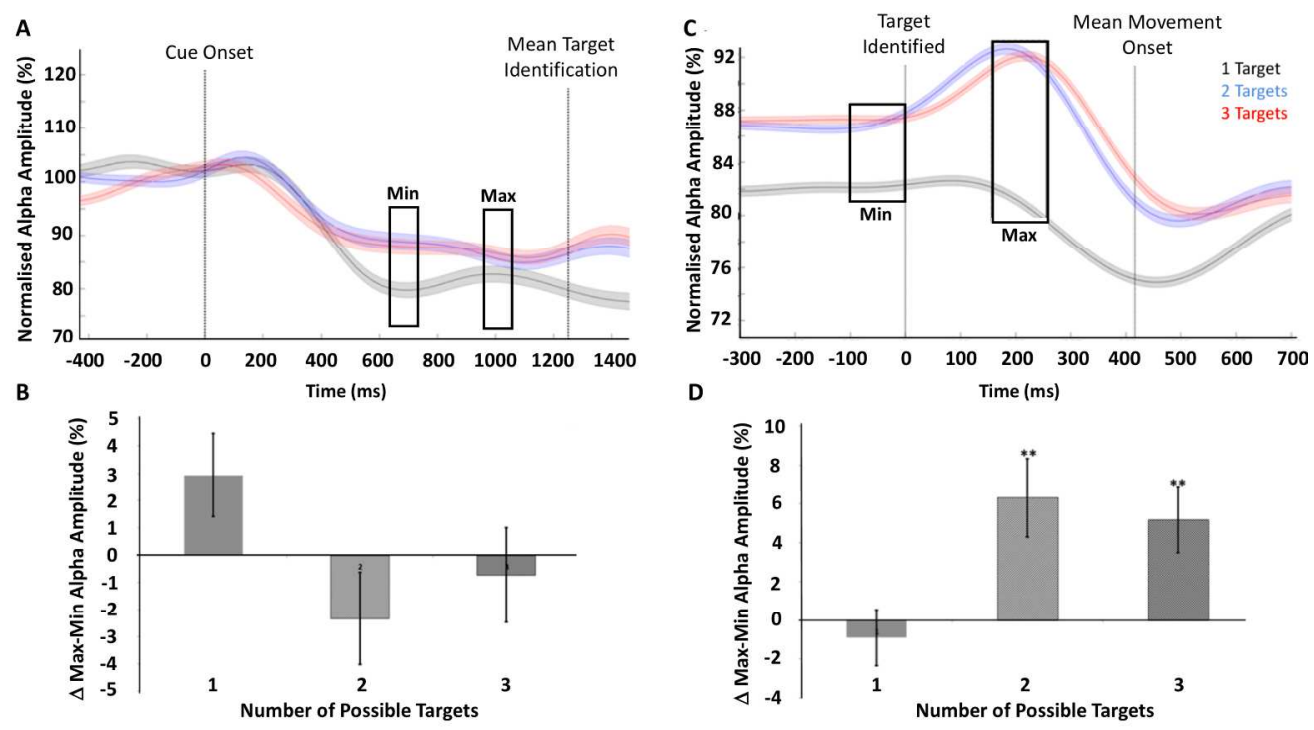

Figure 5. Power envelope plots of normalised alpha power for each of the three target conditions following cue onset and target identification (Mean +/- SEM). (A) Initial changes in preparatory alpha after cue onset. The onset latency ( $<2.5 S D$ of baseline) of alpha ERD did not vary across the three conditions. However, the amplitude of alpha ERD is significantly greater in the 1 target than 2 or 3 target conditions. The black boxes indicate the mean interval of ERD minimum (min) and maximum (max) amplitude in the interval up to target identification. (B) Bar chart showing the change in alpha synchrony (mean +/-SD) between the $100 \mathrm{~ms}$ min and max amplitudes. A non-significant increase in alpha was observed in the 1 target condition but not in the 2 or 3-target conditions, non-significance here is possibly due to the variance across trials as

a result of the length of the interval. (C) Following target identification, the difference in alpha was determined (as denoted by the black boxes) between the $100 \mathrm{~ms}(\mathrm{~min})$ and highest amplitude (max) in the interval between target identification and movement onset. (D) Bar chart showing a significant increase in alpha synchrony (mean +/-SD) following target identification for 2 and 3 target conditions but not the 1 target condition. 